\title{
Nonlinear effects in edge waves
}

\author{
By G. B. WHITHAM \\ Applied Mathematics, California Institute of Technology, Pasadena
}

(Received 2 September 1975)

\begin{abstract}
Nonlinear corrections to Stokes's linear edge-wave solution are obtained by means of perturbation expansions in the amplitude. The shallow-water formulation is considered first, but even for small beach angles $\beta$ the behaviour in the deep water offshore becomes important and this formulation is limited. In the full formulation, amplitude dependence is required in the dispersion relation and in the exponents for the exponential decay away from the shore. There is a nonuniformity in the results as $\beta \rightarrow \frac{1}{2} \pi$, which is corrected by a special perturbation expansion.
\end{abstract}

\section{Introduction}

Stokes (1846) first noted a solution of the linear water-wave equations that represents edge waves on a sloping beach. These are waves propagating along the beach with their crests perpendicular to the shoreline and with amplitude decaying exponentially out to sea. Stokes's solution for the velocity potential $\phi$ and wave height $\zeta$ may be written

$$
\begin{aligned}
& \phi=a g \omega^{-1} \sin \beta \exp (-k y \cos \beta+k z \sin \beta) \sin (k x-\omega t), \\
& \zeta=a \sin \beta \exp (-k y \cos \beta) \cos (k x-\omega t),
\end{aligned}
$$

where $x$ is along the beach, $y$ is out to sea and $z$ is vertical, $\beta$ is the angle of the beach (assumed constant), $a$ is the amplitude of the run-up, and $k$ and $\omega$ are the wavenumber and frequency. The dispersion relation between $\omega$ and $k$ is

$$
\omega^{2}=g k \sin \beta
$$

Munk, Snodgrass \& Carrier (1956) have shown from wave records that edge waves are an important component in the disturbances produced by storms moving along coastlines. They also discuss their generation by diffraction and scattering of incoming wave trains (in particular tsunamis) on irregular and curved coastlines. Apart from such cases where the source provides a component along the beach directly, edge waves may be generated from normally incident waves by instability mechanisms and then play a role in the production of rip currents, scouring of beaches etc. (See Guza \& Davis (1974) and references given there.)

In the further development of the theory, Ursell (1952) showed that Stokes's solution is only one of the possible edge-wave modes and that successively more arise as $\beta$ decreases. A second one is possible for $\beta<\frac{1}{6} \pi$, a third for $\beta<\frac{1}{10} \pi$ 
and so on. The $n$th mode appears as $\beta$ drops below $\pi / 2(2 n+1)$ and its dispersion relation is

$$
\omega^{2}=g k \sin (2 n+1) \beta .
$$

There is also a continuous spectrum of solutions with $\omega^{2}>g k$ to complete the representation of general disturbances. Even in the linear theory, solutions for the continuous spectrum become extremely complicated for arbitrary $\beta$. (See Stoker 1957, chap. 5.) They simplify for the special angles $\beta=\pi / 2 N, N=$ integer (Hanson 1926), but become more complex again as $N$ increases. The case $\beta=1 \pi$ will be useful here and is discussed in detail in $\$ 5$.

When $\beta \ll 1$, it is natural to turn to shallow-water theory. In the linearized version of that theory for a uniformly sloping beach, the surface elevation $\zeta(x, y, t)$ satisfies

$$
g \beta y\left(\zeta_{x x}+\zeta_{y y}\right)+g \beta \zeta_{y}-\zeta_{t t}=0 .
$$

The edge-wave solutions are found from

$$
\begin{gathered}
\zeta=a \beta f(y) \cos (k x-\omega t), \\
y f^{\prime \prime}+f^{\prime}+\left(\omega^{2} / g \beta-k^{2} y\right) f=0 .
\end{gathered}
$$

The equation for $f(y)$ is a slight modification of Laguerre's equation and solutions bounded at both $y=0$ and $y=\infty$ are required. This is a well-known eigenvalue problem with solutions

$$
\begin{aligned}
f(y) & =e^{-k y} L_{n}(2 k y), \\
\omega^{2} & =(2 n+1) \beta g k,
\end{aligned}
$$

where $L_{n}(\xi)$ is the $n$th Laguerre polynomial:

$$
L_{n}(\xi)=e^{\xi}(d / d \xi)^{n}\left(\xi^{n} e^{-\xi}\right) .
$$

The eigenfunctions (8) form a complete set and there is no longer a continuous spectrum. $\uparrow$ The lowest mode, $n=0$, is just

$$
f(y)=e^{-k y}, \quad \omega^{2}=g \beta k,
$$

which agrees with Stokes's result for $\beta \ll 1$. For the higher modes the dispersion relation (9) is the limiting form of Ursell's relation (4). The solutions (8) agree with Ursell's solutions near the shore but there is a discrepancy as $y \rightarrow \infty$. In (8) the asymptotic behaviour is like $y^{n} e^{-k y}$; in Ursell's solutions the decay is pure exponential with slightly modified exponents. This discrepancy is associated with the failure of the shallow-water approximation in the deep water at infinity. The difference is usually unimportant in linear theory since it arises where the disturbance is negligibly small. However, the behaviour at infinity becomes a sensitive issue in the nonlinear theory, and the differences between shallow-water theory and the full theory become important.

The purpose of this paper is to discuss nonlinear effects for travelling edge waves, with emphasis on the nonlinear modifications to the Stokes solution (1)-(3) and its shallow-water counterpart (6) and (11). The first question is

$\dagger$ We are concerned here with the case $k \neq 0$. For $k=0$, edge waves do not arise of course, and there is a continuous spectrum with solutions involving Bessel functions. 
whether solutions representing waves of permanent form exist; the waves could conceivably distort and tend towards breaking under the nonlinear effects, or perhaps swing round towards the shore. The perturbation expansions developed here indicate that waves of permanent form do exist. The appropriate modifications to the linear results for the lowest mode are then found. It appears that similar procedures would go through for the higher modes.

The mathematical problem is beset by a surprising number of non-uniformities. First, amplitude dependence in the dispersion relation is required to avoid secular terms in $x$ or $t$. Fortunately this is familiar in such periodic problems and in fact is the main object of the analysis. Then non-uniformities in the solutions as $y \rightarrow \infty$ become of importance, as noted earlier. These in turn cast doubt on the use of the shallow-water approximation for $\beta \rightarrow 0$, since it is invalid at infinity. The full theory shows that the dispersion relation is given correctly but the $y$ dependence is incorrect. This is analogous to the discrepancy between the linear versions of the two theories, but in severer form since behaviour at infinity enters the arguments directly. Finally, in the full theory there is a non-uniform limit as $\beta \rightarrow \frac{1}{2} \pi$. For a vertical wall $\beta=\frac{1}{2} \pi$, the results should reduce to the known one-dimensional results for deep water (also due to Stokes). They did not. This was a major obstacle. Although the singular behaviour is in a range which is not of much direct interest for edge waves, it left uneasiness about the validity of the other results. A special perturbation solution for $\beta$ near $\frac{1}{2} \pi$ has finally resolved this issue and now all non-uniformities are accounted for.

\section{Shallow-water theory}

We start with the shallow-water theory, since the main steps in the analysis are most easily developed in this simpler formulation.

For a uniformly sloping beach, the nonlinear equations may be written in the form

$$
\begin{aligned}
\zeta_{t}+\left\{(\beta y+\zeta) \Phi_{x}\right\}_{x}+\left\{(\beta y+\zeta) \Phi_{y}\right\}_{y} & =0 \\
\Phi_{t}+\frac{1}{2} \Phi_{x}^{2}+\frac{1}{2} \Phi_{\nu}^{2}+g \zeta & =0
\end{aligned}
$$

where $\Phi(x, y, t)$ is a velocity potential for the horizontal velocity components. For solutions representing travelling edge waves of constant shape, $\zeta$ and $\Phi$ are functions of $\theta=k x-\omega t$ and $y$. To improve on linear theory, $\zeta$ and $\Phi$ are expanded in powers of the run-up amplitude $a$. We take

$$
\begin{aligned}
\zeta & =a \beta\left\{\zeta^{(1)}(\theta, y)+k a \zeta^{(2)}(\theta, y)+k^{2} a^{2} \zeta^{(3)}(\theta, y)+\ldots\right\}, \\
\Phi & =a g \beta \omega^{-1}\left\{\Phi^{(1)}(\theta, y)+k a \Phi^{(2)}(\theta, y)+k^{2} a^{2} \Phi^{(3)}(\theta, y)+\ldots\right\} .
\end{aligned}
$$

As is usual in this type of problem, the possibility of secular terms arises at third order, and a simultaneous expansion of $\omega$ in powers of $a$ is used to suppress them. We take

$$
\omega^{2}=\omega_{0}^{2}\left\{1+\gamma k^{2} a^{2}+\ldots\right\}
$$

a term proportional to $a$ is not required, since there is no trouble in the second approximation. 
The first-order problem leads to the equation

$$
y \zeta_{y y}^{(1)}+\zeta_{y}^{(1)}-\left(\omega_{0}^{2} / g \beta-k^{2} y\right) \zeta_{\theta \dot{y}}^{(1)}=0
$$

for $\zeta^{(1)}$, and $\Phi^{(1)}$ is determined from $\Phi_{\theta}^{(1)}=\zeta^{(1)}$. We choose

$$
\zeta^{(1)}=f(y) \cos \theta, \quad \Phi^{(1)}=f(y) \sin \theta,
$$

in agreement with (6). Then $f(y)$ is one of the eigenfunctions (8) and $\omega_{0}$ is determined by the corresponding eigenvalue relation (9).

At each successive order an equation for $\zeta^{(m)}$ is obtained which is the same as (17) except for inhomogeneous terms on the right involving lower-order quantities. At second order, terms $P(y)+Q(y) \cos 2 \theta$ appear on the right, and the equation may be solved taking a similar form for $\zeta^{(2)}$. At third order we have

$$
y \zeta_{y y}^{(3)}+\zeta_{y}^{(3)}-\left(\omega_{0}^{2} / g \beta-k^{2} y\right) \zeta_{\theta \theta}^{(3)}=R(y) \cos \theta+S(y) \cos 3 \theta
$$

The term in $\cos 3 \theta$ gives no trouble and is accommodated by a corresponding term proportional to $\cos 3 \theta$ in $\zeta^{(3)}$. But the term in $\cos \theta$ would 'resonate' in general, since the solution $f(y) \cos \theta$ chosen for $\zeta^{(1)}$ is a solution of the homogeneous equation. A secular term proportional to $\theta \sin \theta$ would then be required in $\zeta^{(3)}$. However, $R(y)$ includes a term in $\gamma$ which arises from the expansion (16) and has not been determined up to this point. It may be chosen so that resonance does not occur and the appropriate term in $\zeta^{(3)}$ is still $f^{(3)}(y) \cos \theta$. If this is to be the case, we see from (19) that $f^{(3)}$ must satisfy

$$
y f_{y y}^{(3)}+f_{y}^{(3)}+\left(\omega_{0}^{2} / g \beta-k^{2} y\right) f^{(3)}=R(y) .
$$

Now (20) does not have a solution bounded at both $y=0$ and $y=\infty$ for general $R(y)$. This is because the function $f(y)$ in the choice of $\zeta^{(1)}$ is an eigenfunction of the left-hand side with $\omega_{0}^{2} / g \beta$ equal to the corresponding eigenvalue. There is a bounded solution to (20) only when $R(y)$ satisfies the orthogonality condition

$$
\int_{0}^{\infty} f(y) R(y) d y=0 .
$$

The condition may be seen on multiplying (20) by $f(y)$ and integrating by parts to give

$$
\int_{0}^{\infty} f(y) R(y) d y=\left[y\left(f f_{y}^{(3)}-f_{y} f^{(3)}\right)\right]_{0}^{\infty}
$$

If $f^{(3)}$ is bounded at $y=0$ and $y=\infty$, the integrated parts tend to zero (since $f \rightarrow 0$ exponentially as $y \rightarrow \infty$ ) and the orthogonality relation follows.

In simpler problems of this general type, there would be no $y$ dependence in an equation such as (19) and the $\cos \theta$ term would be eliminated completely by the choice of $\gamma$. Here this term is not eliminated completely, but the orthogonality condition is satisfied by the choice of $\gamma$, and a bounded solution for $f^{(3)}$ can be found; there is then no need for secular terms in $\zeta^{(3)}$.

The details of the solution are now given for the lowest (Stokes) mode

$$
f(y)=e^{-k y}, \quad \omega_{0}^{2} / g \beta=k .
$$


This is particularly simple, since the $\cos 2 \theta$ and $\cos 3 \theta$ terms in $\zeta^{(2)}$ and $\zeta^{(3)}$ are absent (by chance) and $\Phi^{(2)}=0$. We find

$$
\zeta^{(2)}=-\frac{1}{2} e^{-2 k y}, \quad \Phi^{(2)}=0 .
$$

Then

$$
\zeta^{(3)}=f^{(3)}(y) \cos \theta, \quad \Phi^{(3)}=f^{(3)}(y) \sin \theta,
$$

where the equation for $f^{(3)}$ is

$$
y f_{y y}^{(3)}+f_{y}^{(3)}+\left(k-k^{2} y\right) f^{(3)}=-\gamma k e^{-k y}+k e^{-3 k y} .
$$

The right-hand side must be orthogonal to $e^{-k y}$; hence $\gamma=\frac{1}{2}$.

This can also be verified directly in the course of solving (25) to find $f^{(3)}$. Since $e^{-k y}$ is a solution of the homogeneous equation, (25) may be solved via the substitution $f^{(3)}=e^{-k y} w(y)$. The equation for $w$ integrates to

$$
w^{\prime}(y)=\frac{1}{4 y}\left(2 \gamma-e^{-2 k y}\right)+\frac{C}{y} e^{2 k y}
$$

For $f^{(3)}$ to be bounded at $\infty, C$ must be zero; for $f^{(3)}$ to be bounded at $y=0, \gamma$ must be $\frac{1}{2}$. Then

$$
f^{(3)}=e^{-k y} w, \quad w=\frac{1}{4} \int_{0}^{y} \frac{1-e^{-2 k \eta}}{\eta} d \eta .
$$

The complete solution to third order is

$$
\begin{aligned}
\zeta & =\beta a\left\{e^{-k y} \cos \theta-\frac{1}{2} k a e^{-2 k y}+k^{2} a^{2} e^{-k y} w(y) \cos \theta+\ldots\right\} \\
\Phi & =g \beta a \omega^{-1}\left\{e^{-k y} \sin \theta+k^{2} a^{2} e^{-k y} w(y) \sin \theta+\ldots\right\}, \\
\omega^{2} & =g k \beta\left(1+\frac{1}{2} k^{2} a^{2}+\ldots\right),
\end{aligned}
$$

the most important result being the nonlinear modification of the dispersion relation in (29).

However, we now come to the non-uniformity as $y \rightarrow \infty$. Although $f^{(3)}$ is bounded and in fact tends to zero as $y \rightarrow \infty$, it becomes large compared with $e^{-k y}$, since

$$
w(y) \sim \frac{1}{4} \log y, \quad y \rightarrow \infty .
$$

Therefore the third-order terms in (27) and (28) become large compared with the first terms and the expansions in $k a$ are not uniformly valid as $y \rightarrow \infty$. When this occurs in a perturbation expansion it is normally viewed as an inadvertent use of a Taylor series which should be reversed. That is, the first and third terms in (27) should be combined into

$$
\exp \left[-k y+k^{2} a^{2} w(y)\right] \cos \theta ;
$$

we have found the Taylor expansion

$$
e^{-k y}\left\{1+k^{2} a^{2} w(y)+\ldots\right\} \cos \theta .
$$

Since $w / y$ remains bounded for all $y$ (and in fact tends to zero as $y \rightarrow \infty$ ), (31) would be proposed as the uniformly valid form.

The analysis can be recast to incorporate (31). This leads naturally to a revised view of the expansions for $\zeta$ and $\Phi$, in which the terms are regrouped according to Fourier expansions. That is, we take

$$
\begin{aligned}
\zeta & =\beta a\left\{k a f_{0}(y, a)+f_{1}(y, a) \cos \theta+k a f_{2}(y, a) \cos 2 \theta+k^{2} a^{2} f_{3}(y, a) \cos 3 \theta+\ldots\right\} \\
\Phi & =g \beta a \omega^{-1}\left\{g_{1}(y, a) \sin \theta+k a g_{2}(y, a) \sin 2 \theta+k^{2} a^{2} g_{3}(y, a) \sin 3 \theta+\ldots\right\} .
\end{aligned}
$$


The powers of $k a$ indicate the main orders of the terms, but further amplitude dependence remains in the coefficients. If the coefficients have Taylor expansions in $k a$, then after re-arrangement the previous version is recovered. However, the Fourier-series form has the greater flexibility required to incorporate (31). The details are not pursued in this case because the shallow-water approximation is now suspect. The behaviour at infinity becomes a crucial part of the discussion, and is also involved in deriving the dispersion relation (29) (from the condition that $f^{(3)}$ be bounded at $\infty$ ). Since the shallow-water assumptions break down in the deep water at infinity it is not clear even that (29) is correct. Certainly (31) has an awkward form when (26) is used for $w(y)$. We also note that $w(y) \sim \frac{1}{4} \log y$ so the $y$ dependence is like

$$
y^{\frac{1}{4} k^{2} a^{2}} e^{-k y} \quad \text { as } \quad y \rightarrow \infty \text {. }
$$

This is reminiscent of the comparison made following (11) of the linear results. The conclusion is that (31) is not the true behaviour in the real problem. Accordingly the full formulation is taken up. It is found that the dispersion relation (29) is correct for $\beta \rightarrow 0$, but the behaviour in (31) should again be pure exponential in $y$, with an exponent depending on $k a$.

A. Minzoni has suggested that the shallow-water results could be clarified by considering a more general depth distribution $h_{0}(y)$ which starts like $\beta y$ but remains finite and shallow as $y \rightarrow \infty$. His investigation appears in the adjacent paper. He finds results similar to those of the full theory. The case $h_{0}=\beta y$ is indeed anomalous in shallow-water theory.

\section{Fourier series expansions for the full water wave theory}

In the full theory, the problem is formulated in terms of a velocity potential $\phi(x, y, z, t)$ which satisfies Laplace's equation in the wedge-shaped region bounded by $z=0$ and $y \sin \beta+z \cos \beta=0$. The normal derivative

$$
\phi_{y} \sin \beta+\phi_{z} \cos \beta=0
$$

on the sloping side, and there are nonlinear boundary conditions relating $\phi$ and the wave height $\zeta(x, y, t)$ on $z=0$.

In view of the various non-uniformities we take the Fourier-series view from the start. The amplitude expansions can be recovered as described at the end of the last section. The Fourier expansions are

$$
\begin{aligned}
& \phi=a g \omega^{-1} \sin \beta\left\{F(y, z, a) \sin \theta+k a F_{2}(y, z, a) \sin 2 \theta\right. \\
&\left.+k^{2} a^{2} F_{3}(y, z, a) \sin 3 \theta+\ldots\right\}, \\
& \zeta=a \sin \beta\left\{k a G_{0}(y, a)+G(y, a) \cos \theta+\right. k a G_{2}(y, a) \cos 2 \theta \\
&\left.+k^{2} a^{2} G_{3}(y, a) \cos 3 \theta+\ldots\right\},
\end{aligned}
$$

where $\theta=k x-\omega t$, and $\omega(k, a)$ is not expanded at this stage. For Stokes's edge wave the boundary-value problem for $F$ correct to third order is

$$
\begin{gathered}
F_{y y}+F_{z z}-k^{2} F=0, \quad-y \tan \beta<z<0, \\
F_{z}-\left(\omega^{2} / g\right) F=-k^{3} a^{2} \sin \beta F^{3}, \quad z=0, \\
F_{y} \sin \beta+F_{z} \cos \beta=0, \quad z=-y \tan \beta .
\end{gathered}
$$

The derivation will be explained after noting the linear solution. 
In the linearized theory the right-hand side of (38) is neglected and Stokes's edge wave

$$
\begin{gathered}
F=\exp (-k y \cos \beta+k z \sin \beta), \\
\omega^{2} / g=k \sin \beta
\end{gathered}
$$

is a solution. The corresponding expression for $G$ is

$$
G=F(y, 0)=\exp (-k y \cos \beta) .
$$

The nonlinear problem is obtained by substituting (35) and (36) into the governing equations. Laplace's equation gives (37) for $F$ and

$$
F_{n y y}+F_{n z z}-n^{2} k^{2} F_{n}=0
$$

for each $F_{n}$. All the $F$ 's satisfy (39), since the bottom condition (34) is linear and does not involve $\theta$ derivatives. The nonlinear boundary conditions on the top surface $z=0$ are complicated. After substitution of (35) and (36), the various products are re-expressed as Fourier series, keeping terms that contribute up to third order. The coefficients of the combined Fourier series are then set individually to zero. Now it is anticipated that the corrections to the linear relations are of order $a^{2}$. So we use the relations

$$
\begin{gathered}
F_{y}=-k \cos \beta F+O\left(a^{2}\right), \quad F_{z}=k \sin \beta F+O\left(a^{2}\right), \\
G=F+O\left(a^{2}\right), \quad \omega^{2}=g k \sin \beta+O\left(a^{2}\right)
\end{gathered}
$$

in any second- and third-order quantities. For example, the terms independent of $\theta$ in one of the boundary conditions give

$$
G_{0}=\frac{1}{2 k} \sin \beta F_{z} G-\frac{1}{4} \frac{g \sin \beta}{\omega^{2} k}\left(k^{2} F^{2}+F_{y}^{2}+F_{z}^{2}\right) .
$$

From (43) and (44) we have

$$
G_{0}=-\frac{1}{2} \cos ^{2} \beta F^{2}+O\left(a^{2}\right),
$$

which is sufficient for third-order accuracy in (36). Similarly it is found from the terms in $\cos 2 \theta$ and $\sin 2 \theta$ that

$$
G_{2}=\frac{1}{2} \sin ^{2} \beta F^{2}, \quad F_{2}=0
$$

to this order. Third-order products contribute to both the first and third harmonics, since, for example, $\cos ^{3} \theta$ and $\cos \theta \cos 2 \theta$ may be re-expressed as combinations of $\cos \theta$ and $\cos 3 \theta$. When (46) and (47), as well as (43) and (44), are used in their coefficients, we obtain

$$
\left.\begin{array}{c}
G_{3}=\frac{3}{8} \sin ^{4} \beta F^{3}, \quad F_{3}=0, \\
=F-k^{2} a^{2}\left(\frac{3}{2} \sin ^{2} \beta-\frac{9}{8} \sin ^{4} \beta\right) F^{3}
\end{array}\right\}
$$

and the boundary condition (38).

A detailed derivation is given in the appendix. 


\section{The boundary-value problem for $F$}

The first approach to the boundary-value problem (37)-(39) is to use small amplitude expansions to correct the Stokes solution, i.e.

$$
\begin{gathered}
F=\exp (-k y \cos \beta+k z \sin \beta)+k^{2} a^{2} F^{(3)}(y, z)+\ldots, \\
\omega^{2} / g=k \sin \beta\left(1+\gamma k^{2} a^{2}+\ldots\right) .
\end{gathered}
$$

Then

$$
F_{y y}^{(3)}+F_{z z}^{(3)}-k^{2} F^{(3)}=0, \quad-y \tan \beta<z<0,
$$

$$
\begin{gathered}
F_{z}^{(3)}-k \sin \beta F^{(3)}=k \sin \beta\{\gamma \exp (-k y \cos \beta)-\exp (-3 k y \cos \beta)\}, \quad z=0, \\
F_{y}^{(3)} \sin \beta+F_{z}^{(3)} \cos \beta=0, \quad z=-y \tan \beta .
\end{gathered}
$$

This is exactly the problem that would have been obtained for the resonant term in the more straightforward amplitude expansions for $\phi$ and $\zeta$. It is the analogue of (25). There is again no bounded solution for the inhomogeneous problem in general since

$$
\psi=\exp (-k y \cos \beta+k z \sin \beta)
$$

is an eigensolution of the homogeneous problem. But an orthogonality condition analogous to that for (25) can be found and when this is satisfied there is a bounded solution for $F^{(3)}$. The condition can be obtained by applying Green's theorem to $\psi$ and $F^{(3)}$ over the wedge-shaped region. We have

$$
\begin{aligned}
0 & =\iint\left(\psi \nabla^{2} F^{(3)}-F^{(3)} \nabla^{2} \psi\right) d y d z \\
& =\int_{0}^{\infty}\left[\psi F_{z}^{(3)}-F^{(3)} \psi_{z}\right]_{z=0} d y \\
& =\int_{0}^{\infty} \exp (-k y \cos \beta)\left[F_{z}^{(3)}-k \sin \beta F^{(3)}\right]_{z=0} d y .
\end{aligned}
$$

Without the extra term containing $\gamma$ in (52), the condition could not be satisfied and there would be no solution for $F^{(3)}$. When it is included, we have a simple determination of $\gamma$; it must be $\frac{1}{2}$. This confirms the result (29) of shallow-water theory in the limit as $\beta \rightarrow 0$.

At first this seemed a neat end to the investigation and it was hoped that this time there would be no trouble at infinity. But it was soon noted that, in the limit $\beta \rightarrow \frac{1}{2} \pi$, the nonlinear correction in (50) with $\gamma=\frac{1}{2}$ does not reduce to Stokes's result for a one-dimensional wave in deep water. (A vertical wall parallel to the direction of propagation has no effect in inviscid theory.) In that case $\gamma=1$ ! Indeed for $\beta=\frac{1}{2} \pi$ and $y$ dependence absent, the solution of (51)-(53) is clearly just

in agreement with Stokes.

$$
F^{(3)}=e^{k z}, \quad \gamma=1,
$$

Now the question of non-uniformity as $y \rightarrow \infty$ becomes an issue again. So far $F^{(3)}$ has not been determined. Does it, in fact, have highly unacceptable properties which invalidate the whole discussion?

This was first resolved by finding $F^{(3)}$ in detail for the special case $\beta=\frac{1}{4} \pi$. But the consequences can be put in general form. For large $y$, we argue that the 
term $\exp (-3 k y \cos \beta)$ in (52) is negligible (provided $\beta$ is not close to $\left.\frac{1}{2} \pi !\right)$ and that the waves no longer feel the bottom so (53) can be ignored. Then a solution of (51) and the approximate form of (52) with $\gamma=\frac{1}{2}$ is

$$
F^{(3)} \sim\left(\frac{1}{2} k y \sin ^{2} \beta / \cos \beta+\frac{1}{2} k z \sin \beta\right) \exp (-k y \cos \beta+k z \sin \beta) .
$$

When this is substituted back in (49) we have again non-uniform behaviour at infinity. However, this time, reversing the Taylor expansion, the proposed uniformly valid form is

where

$$
F \sim \exp (-k A y+k B z),
$$

This seems perfectly reasonable; we just have amplitude dependence in the exponents. The frequency $\omega$ already had to depend on $a$; it seems natural that other parameters should also.

However, non-uniformity as $\beta \rightarrow \frac{1}{2} \pi$ also appears. Clearly the correction term in (57) is not valid in this limit, and we know in any case that (50) is not valid with $\gamma=\frac{1}{2}$. The conclusion is that amplitude dependence of $\omega, A$ and $B$ is correct but the corrections can not be obtained by simple expansions in $a$ for $\beta$ close to $\frac{1}{2} \pi$.

The validity of $(56)-(58)$ for $\beta$ well away from $\frac{1}{2} \pi$ will be discussed in $\S 6$ and the behaviour as $\beta \rightarrow \frac{1}{2} \pi$ will be obtained in $\S 7$. First we justify the result (55) and the arguments leading to it for $\beta=\frac{1}{4} \pi$.

\section{Special case $\beta=\frac{1}{4} \pi$}

The solution of the boundary-value problem (51)-(53) is now explored in full for the case $\beta=\frac{1}{4} \pi$. We shall need the solution of the homogeneous eigenvalue problem

$$
\begin{aligned}
\psi_{y y}+\psi_{z z}-k^{2} \psi & =0, \quad-y<z<0 \\
\psi_{z}-\lambda \psi=0, & z=0 \\
\psi_{y}+\psi_{z} & =0, \quad z=-y .
\end{aligned}
$$

The edge wave $\quad \psi_{0}=\exp \left(-\lambda_{0} y+\lambda_{0} z\right), \quad \lambda_{0}=k / \sqrt{ } 2$,

is one solution. The solution in the continuous spectrum may be written as

$$
\begin{gathered}
\psi_{l}=\frac{1}{2}(l+i \lambda) \exp (i l y+\lambda z)+\frac{1}{2}(l+i \lambda) \exp (-\lambda y-i l z)+\text { c.c. } \\
\lambda=\left(l^{2}+k^{2}\right)^{\frac{1}{2}} \quad \text { with } \quad 0<l<\infty
\end{gathered}
$$

where c.c. denotes 'complex conjugate'. The first term in (63) is the usual oblique wave for infinite depth; it satisfies the condition on $z=0$ but not that on $z=-y$. The second term compensates in order to satisfy the condition on $z=-y$; it falls off as $y \rightarrow \infty$. (Notice that this term must be combined with its complex conjugate to satisfy the condition on $z=0$.)

The solution (63)-(64) was found by Hanson (1926), presumably by trying exponential solutions and choosing parameters appropriately. It can also be 
derived by an extension of a method used by Stoker (1957, chap. 5) for beach angles which are fractions of $\frac{1}{2} \pi$.

In the inhomogeneous problem for $F^{(3)},(60)$ is replaced by

$$
F_{z}^{(3)}-\lambda_{0} F^{(3)}=\lambda_{0}\left(\gamma e^{-\lambda_{0} y}-e^{-3 \lambda_{0} y}\right), \quad z=0 .
$$

(It is convenient to use $\lambda_{0}$ to avoid writing $k / \sqrt{ } 2$ everywhere.) The solution for $F^{(3)}$ is found as a superposition of the eigensolutions of the homogeneous problem. We take

$$
F^{(3)}(y, z)=F_{0} \psi_{0}(y, z)+\int_{0}^{\infty} F_{l} \psi_{l}(y, z,) d l .
$$

This satisfies (51) and (53) immediately and the coefficients $F_{0}$ and $F_{l}$ are to be determined from (65). We shall need the expansion of the right-hand side in the reduced eigenfunctions

$$
\Psi_{l}(y) \equiv \psi_{l}(y, 0)=\frac{1}{2}(l+i \lambda) e^{i l y}+\frac{1}{2}(l+i \lambda) e^{-\lambda y}+\text { c.c. }
$$

These are orthogonal and any square-integrable function $f(y)$ can be expanded as

where

$$
f(y)=f_{0} e^{-\lambda_{0} y}+\int_{0}^{\infty} f_{l} \Psi_{l}(y) d l
$$

$$
f_{l}=\frac{2}{\pi\left(\lambda^{2}+l^{2}\right)} \int_{0}^{\infty} f(y) \Psi_{l}(y) d y, \quad \lambda=\left(l^{2}+k^{2}\right)^{\frac{1}{2}} .
$$

For $f(y)=\lambda_{0}\left(\gamma e^{-\lambda_{0} y}-e^{-3 \lambda_{0} y}\right)$, we have

$$
f_{0}=\lambda_{0}\left(\gamma-\frac{1}{2}\right), \quad f_{l}=-\frac{16 \lambda_{0}^{2} l}{\pi\left(l^{2}+\lambda_{0}^{2}\right)\left(l^{2}+9 \lambda_{0}^{2}\right)\left(\lambda+3 \lambda_{0}\right)} .
$$

When (66) is substituted in (65), the term $\Psi_{0}$ drops out on the left so it must be absent on the right. This is another derivation of the orthogonality condition, namely $f_{0}=0$, and the requirement $\gamma=\frac{1}{2}$ is recovered. From the continuous range, we find

$$
F_{l}=f_{l} /\left(\lambda-\lambda_{0}\right) \text {. }
$$

When $F_{l}$ and $\psi_{l}$ are substituted in (66), the complex-conjugate part can be converted into an integral over negative $l$ and the final solution written as

$$
F^{(3)}=-\frac{8 \lambda_{0}^{2}}{\pi} \int_{-\infty}^{\infty} \frac{l(l+i \lambda)\left\{e^{i l y+\lambda z}+e^{-\lambda y-i l z}\right\} d l}{\left(l^{2}+\lambda_{0}^{2}\right)\left(l^{2}+9 \lambda_{0}^{2}\right)\left(\lambda+3 \lambda_{0}\right)\left(\lambda-\lambda_{0}\right)}
$$

In the upper half-plane there is a double pole at $l=i \lambda_{0}\left(\lambda=\lambda_{0}\right)$, a single pole at $l=3 i \lambda_{0}$ and a branch point at $l=i k(\lambda=0)$. If the plane is cut from $l=i k$ to $i \infty$ and $\mathscr{C}$ is a loop contour around the cut, the path of integration for the $e^{i l+\lambda \varepsilon}$ term in (73) can be deformed around the pole $l=i \lambda_{0}$ into $\mathscr{C}$, to give

$$
\begin{gathered}
F^{(3)}=\frac{1}{2} \lambda_{0}(y+z) e^{-\lambda_{0} y+\lambda_{0} z}+H, \\
H=-\frac{8 \lambda_{0}^{3}}{\pi} \int_{\mathscr{C}} \frac{l(l+i \lambda) e^{i l y+\lambda z} d l}{\left(l^{2}+\lambda_{0}^{2}\right)\left(l^{2}+9 \lambda_{0}^{2}\right)\left(\lambda+3 \lambda_{0}\right)\left(\lambda-\lambda_{0}\right)} \\
-\frac{8 \lambda_{0}^{3}}{\pi} \int_{-\infty}^{\infty} \frac{l(l+i \lambda) e^{-\lambda y-i l z} d l}{\left(l^{2}+\lambda_{0}^{2}\right)\left(l^{2}+9 \lambda_{0}^{2}\right)\left(\lambda+3 \lambda_{0}\right)\left(\lambda-\lambda_{0}\right)}
\end{gathered}
$$


The two integrals can be transformed and combined in various interesting ways, but all we need note here is that they are both $O\left(e^{-k v y}\right)$, whereas the first term in (74) is $O\left(e^{-k y / \sqrt{ } 2}\right)$. The first term is exactly $(55)$ for the case $\beta=\frac{1}{4} \pi$. It satisfies the part

$$
F_{z}^{(3)}-\lambda_{0} F^{(3}=\frac{1}{2} \lambda_{0} e^{-\lambda_{0} y}, \quad z=0,
$$

of (65) and Laplace's equation, but not the bottom condition. The function $H$ balances the remaining term in (65) and ensures satisfaction of the boundary condition at the bottom.

Thus the arguments leading to (55) are justified in all details for the special case $\beta=\frac{1}{4} \pi$. It seems clear that they hold in general provided $\beta$ is not too close to $\frac{1}{2} \pi$.

\section{Revised solution of the boundary-value problem}

The solution proposed in (56) is a modification of the linear solution to allow the exponents as well as $\omega$ to depend upon $a$. To assess this directly in (37)-(39) we consider the expansions

$$
\begin{gathered}
F=e^{-k A y+k B z}+k^{2} a^{2} H+\ldots, \\
\omega^{2} / g=k \sin \beta\left(1+\gamma k^{2} a^{2}+\ldots\right), \\
A=\cos \beta\left(1+A_{2} k^{2} a^{2}+\ldots\right), \quad B=\sin \beta\left(1+B_{2} k^{2} a^{2}+\ldots\right) .
\end{gathered}
$$

The first term in $F$ must satisfy (37), so that

$$
A^{2}+B^{2}=1 \text {, i.e. } A_{2} \cos ^{2} \beta+B_{2} \sin ^{2} \beta=0,
$$

and the boundary-value problem for $H$ becomes

$$
\begin{gathered}
H_{y y}+H_{z z}-k^{2} H=0, \quad-y \tan \beta<z<0, \\
H_{z}-k \sin \beta H=k \sin \beta\left\{\left(\gamma-B_{2}\right) e^{-k A y}-e^{-3 k A y}\right\}, \quad z=0, \\
H_{y} \sin \beta+H_{z} \cos \beta=k \sin \beta \cos \beta\left(A_{2}-B_{2}\right) e^{-k r}, \quad z=-y \tan \beta ;
\end{gathered}
$$

in the last term, $r$ is the radial distance $\left(y^{2}+z^{2}\right)^{\frac{1}{2}}$. It should be noted that the relations (43) and (44) used to express the higher-order terms in $F$ still hold to the order shown.

Now the previous arguments can be applied to the revised problem. First, from (81) we choose

to avoid a term of the form

$$
\gamma=B_{2}
$$

$$
(p y+q z) e^{-k \Delta y+k B z},
$$

which would lead to a non-uniform expansion in (76). Then we derive the revised orthogonality condition. It has an extra term since $\partial H / \partial n$ is no longer zero on the bottom. It reads

$$
\int_{0}^{\infty}\left[H_{z}-k \sin \beta H\right]_{z=0} e^{-k y \cos \beta} d y-\int_{0}^{\infty}\left[H_{y} \sin \beta+H_{z} \cos \beta\right]_{z=-y \tan \beta} e^{-k r} d r=0 .
$$

This gives

$$
A_{2}-B_{2}=-\frac{1}{2} \sec ^{2} \beta
$$

in the lowest-order approximation. 
The coefficients $\gamma, A_{2}$ and $B_{2}$ are determined from (79), (83) and (85) and we have

$$
\begin{aligned}
\omega^{2} & =g k \sin \beta\left(1+\frac{1}{2} k^{2} a^{2}+\ldots\right) \\
A & =\cos \beta-\frac{1}{2} k^{2} a^{2} \sin ^{2} \beta / \cos \beta+\ldots, \\
B & =\sin \beta+\frac{1}{2} k^{2} a^{2} \sin \beta+\ldots
\end{aligned}
$$

With these conditions satisfied, it is assumed that there is a satisfactory solution for $H$ (when $\beta \neq \frac{1}{2} \pi$ ) and the second term in (76) is uniformly small. This can be checked for the case $\beta=\frac{1}{4} \pi$. In fact, if the linear value $A=1 / \sqrt{ } 2$ is taken in the exponent in (81) the solution is just (75). Otherwise some care is needed (in particular the orthogonality relation must be satisfied more accurately than in (85)) but the modifications are inconsequential and do not affect (86)-(88) to the order shown.

The important results are the first term in (76) and the expressions for the parameters in $(86)-(88)$. These agree with $(56)-(58)$ and provide formal justification. The nonlinear effects reduce the exponential decay with $y$ and introduce amplitude dependence into the dispersion relation. The non-uniformity as $\beta \rightarrow \frac{1}{2} \pi$ is apparent in both the results and the derivation. In a small range $\frac{1}{2} \pi-\beta=O(k a)$ near to $\frac{1}{2} \pi$, the two terms in (87) are of equal order. In fact if the formula still held, $A$ would reach zero at a value in this range before $\beta$ reached $\frac{1}{2} \pi$. This indicates that the simple exponential decay ceases before $\beta$ reaches $\frac{1}{2} \pi$. Similarly $B$ is increased over its linear value and reaches unity before $\beta=\frac{1}{2} \pi$; from (79), $B$ can not exceed unity. In the derivation it is clear that as $A \rightarrow 0$ the terms on the right of (81) become equally important and the argument used to determine $\gamma$ is invalid when this occurs. The exact solution of (37)-(39) for $\beta=\frac{1}{2} \pi$ is

$$
F=e^{k z}, \quad \omega^{2} / g k=1+k^{2} a^{2},
$$

so we do have $A=0, B=1$ and a different value for $\gamma$ in the limit. All this indicates that (76) and (86)-(88) are valid provided that $k a / \cos \beta$ is small, and that there is a rapid transition in the range $\frac{1}{2} \pi-\beta=O(k a)$ to the solution (89). A perturbation procedure is now developed for this transition range.

\section{The limit $\beta \rightarrow \frac{1}{2} \pi$}

When $\frac{1}{2} \pi-\beta=O(k a), \cos \beta=O(k a)$ and we have

$$
A=O(k a), \quad B=1+O\left(k^{2} a^{2}\right), \quad \omega^{2} / g k=1+O\left(k^{2} a^{2}\right) .
$$

The first two of these are interpreted as

$$
\begin{gathered}
F_{y}=O\left(k^{2} a F\right), \\
F=e^{k z} U, \text { where } \quad U_{\bar{z}}=O\left(k^{3} a^{2} U\right) .
\end{gathered}
$$

A change of variables that accommodates these orders of magnitude is

$$
\begin{array}{cl}
\cos \beta=\alpha k a, & \omega^{2} / g k=1+\mu k^{2} a^{2} \\
F=e^{k z} U(Y, Z), \quad Y=k^{2} a y, \quad Z=k^{3} a^{2} z
\end{array}
$$


When these are introduced into (37)-(39), and the limit $k a \rightarrow 0$ taken in the resulting equations, the reduced boundary-value problem is

$$
\begin{gathered}
U_{Y Y}+2 U_{Z}=0, \quad-Y<\alpha Z<0, \\
U_{Z}-\mu U=-U^{3}, \quad Z=0, \\
U_{Y}+\alpha U=0, \quad Y=-\alpha Z .
\end{gathered}
$$

On $Z=0, U_{Z}$ can be eliminated from (94) using (93) and we have the eigenvalue problem

$$
\begin{gathered}
u^{\prime \prime}+2 \mu u=2 u^{3}, \quad 0<Y<\infty, \\
u^{\prime}+\alpha u=0, \quad Y=0, \\
u \text { bounded, } \quad Y=\infty
\end{gathered}
$$

for $\mu$ and $u(Y)=U(Y, 0)$. It is remarkable that the surface conditions are decoupled in this way. The amplitude $a$ can be defined such that $F=1$ at the origin, then the boundary condition at $Y=0$ may be taken as

$$
u=1, \quad u^{\prime}=-\alpha, \quad Y=0 .
$$

Equation (96) integrates to

$$
u^{\prime 2}+2 \mu u^{2}=u^{4}+C .
$$

There are now two cases depending on whether or not $u \rightarrow 0$ as $Y \rightarrow \infty$.

Case I: $u \rightarrow 0, Y \rightarrow \infty$. In this case $C=0$ and $\mu$ must be negative. The boundary conditions (99) then determine $\mu$ to be

The solution is

$$
\mu=-\frac{1}{2}\left(\alpha^{2}-1\right) \text { for } \alpha>1
$$

$$
u=\frac{2 c}{(1+c) \exp \left[Y\left(\alpha^{2}-1\right)^{\frac{1}{2}}\right]-(1-c) \exp \left[-Y\left(\alpha^{2}-1\right)^{\frac{1}{2}}\right]}, \quad c=\frac{\left(\alpha^{2}-1\right)^{\frac{1}{2}}}{\alpha} .
$$

When $\alpha \gg 1$,

$$
u \sim \exp \left[-Y\left(\alpha^{2}-1\right)^{\frac{1}{2}}\right]+O\left\{\alpha^{-2} \exp \left[-3 Y\left(\alpha^{2}-1\right)^{\frac{1}{2}}\right]\right\} .
$$

In the original variables this is the range $\cos \beta \gg k a$, and (101) and (103) give

$$
\begin{gathered}
\omega^{2} / g k \sim 1-\frac{1}{2} \cos ^{2} \beta+\frac{1}{2} k^{2} a^{2}, \\
F(y, 0) \sim e^{-k A y}+O\left(k^{2} a^{2} \cos ^{-2} \beta e^{-3 k A y}\right), \\
A=\left(\alpha^{2}-1\right)^{\frac{1}{2}} k a \sim \cos \beta-\frac{1}{2} k^{2} a^{2} / \cos \beta .
\end{gathered}
$$

where

These agree with (86) and (87) when $\cos \beta \ll 1$. (Note that $\sin \beta \sim 1-\frac{1}{2} \cos ^{2} \beta$.) Therefore this perturbation solution merges correctly with the previous solution in the range $k a \ll \cos \beta \ll 1$.

Case I applies when $\alpha>1$, i.e. $\cos \beta>k a$, but ceases to give a bounded solution otherwise. This means that the solution no longer tends to zero as $Y \rightarrow \infty$, when $\alpha<1$, i.e. $\cos \beta<k a$. We then have the following.

Case II: $u \rightarrow b>0, Y \rightarrow \infty$. In this case

$$
u^{\prime 2}=C-2 \mu u^{2}+u^{4}
$$


and $u=b$ must be a double zero of the right-hand side. Hence $\mu=b^{2}$ and $C=b^{4}$. The boundary conditions (99) then require

$$
\begin{gathered}
b^{2}=1-\alpha, \\
\mu=1-\alpha, \quad \alpha<1 .
\end{gathered}
$$

The solution is

$$
u=b\left\{\frac{(1+b)+(1-b) e^{-2 b F}}{(1+b)-(1-b) e^{-2 b Y}}\right\} .
$$

Therefore, in this range, $0<\cos \beta<k a$,

$$
\omega^{2} / g k=1+(k a-\cos \beta) k a,
$$

and $F$ decreases from 1 at $y=0$ to

$$
b=(1-\cos \beta / k a)^{\frac{1}{2}} \quad \text { as } \quad y \rightarrow \infty .
$$

When $\cos \beta=0$, we have the deep-water result (89), and everything checks.

With $U(Y, 0)$ determined, the solution of (93) and (95) for the $Z$ dependence is now a linear problem. If new variables

$$
Y+\alpha Z=\eta, \quad Z=-\zeta
$$

are introduced the problem becomes

$$
\begin{gathered}
U_{\eta \eta}+2 \alpha U_{\eta}=U_{\zeta}, \quad \eta>0, \quad \zeta>0, \\
U_{\eta}+\alpha U=0, \quad \eta=0, \\
U=u(\eta), \quad \zeta=0 .
\end{gathered}
$$

The solution can be obtained via Laplace transforms in $\zeta$. The details will not be given, since the interesting results concerning the behaviour at the surface have already been found.

\section{The dispersion relation and modulation theory}

The dispersion relation controls the behaviour and stability of modulated wave trains (see Whitham 1974, chaps 14 and 15). For a wave train with

$$
\omega=\omega_{0}(k)+\omega_{2}(k) a^{2}+\ldots
$$

the criterion for instability in the sense that small modulations will grow is $\omega_{0}^{\prime \prime} \omega_{2}<0$. Here, in all cases (including Stokes's deep-water solution (89)), $\omega_{0}^{\prime \prime}<0$ and $\omega_{2}>0$, so we have the unstable situation. The inference from similar problems is that this kind of instability does not lead to chaotic motion, but rather to a tendency for concentrated groups to form. The local behaviour inside a group is given by the periodic solution but the amplitude and frequency are modulated into the form typical of solitary waves. In simpler problems (Whitham 1974, $\S 15.5,17.8$; Ostrowskii 1967, 1968) such 'solitary envelopes' can be described in detail. The detailed description here would be formidable.

This research was supported by the Office of Naval Research, U.S. Navy. 


\section{Appendix}

The boundary conditions on the free surface $z=\zeta$ are

$$
\begin{aligned}
& \zeta_{t}+\phi_{x} \zeta_{x}+\phi_{y} \zeta_{y}=\phi_{z}, \\
& \phi_{t}+\frac{1}{2}(\nabla \phi)^{2}+g \zeta=0 .
\end{aligned}
$$

These may be transferred to $z=0$ using Taylor expansions in $\zeta$. To third order they become

$$
\begin{aligned}
\zeta_{t}-\phi_{z}+\left\{\phi_{x} \zeta_{x}+\phi_{y} \zeta_{y}-\phi_{z z} \zeta\right\}+\left[\phi_{x z} \zeta \zeta_{x}+\phi_{y z} \zeta \zeta_{y}-\frac{1}{2} \phi_{z z z} \zeta^{2}\right] & =0 \\
\phi_{t}+g \zeta+\left\{\frac{1}{2}(\nabla \phi)^{2}+\phi_{t z} \zeta\right\}+\left[\frac{1}{2}(\nabla \phi)_{z}^{2} \zeta+\frac{1}{2} \phi_{t z z} \zeta^{2}\right] & =0
\end{aligned}
$$

The second one can be solved for $\zeta$ and used in the first to find a boundary condition for $\phi$ alone. By hindsight a grouping of the terms can be found that makes the final algebra simple. The guide is to use $(\nabla \phi)^{2}$ and $\phi_{z}+g^{-1} \phi_{t t}$ as much as possible; the latter is the linearized boundary condition. Then it is found that

$$
\begin{aligned}
\phi_{z}+\frac{1}{g} \phi_{t t}=-\frac{1}{g}(\nabla \phi)_{t}^{2}+ & \left\{\frac{1}{g} \phi_{t}\left(\phi_{z}+\frac{1}{g} \phi_{t t}\right)\right\}_{z}-\frac{1}{2 g}\left[\phi_{x}(\nabla \phi)_{x}^{2}+\phi_{y}(\nabla \phi)_{y}^{2}+\phi_{z}(\nabla \phi)_{z}^{2}\right] \\
& +\left[\frac{1}{g^{2}} \phi_{t}(\nabla \phi)_{t}^{2}\right]_{z}+\left[\frac{1}{2 g}\left(\phi_{z}+\frac{1}{g} \phi_{t t}\right)\left((\nabla \phi)^{2}-\frac{1}{g} \phi_{t}^{2}\right)\right]_{z} .
\end{aligned}
$$

The Fourier series for $\phi$ is

$$
\phi=a g \omega^{-1} \sin \beta\left\{F \sin \theta+k a F_{2} \sin 2 \theta+k^{2} a^{2} F_{3} \sin 3 \theta+\ldots\right\},
$$

where $\theta=k x-\omega t$. In principle, terms quadratic in $F$ on the right of (A 3) generate $F_{2}$; cubic terms then generate $F_{3}$ as well as contributing to the first harmonic. However, the first-order results

$$
F_{y}=-k \cos \beta F, \quad F_{z}=k \sin \beta F, \quad \omega^{2}=g k \sin \beta
$$

may be used on the right of (A 3), and with these approximations

$$
(\nabla \phi)^{2}=g^{2} a^{2} \omega^{-2} \sin ^{2} \beta k^{2} F^{2}, \quad \phi_{z}+g^{-1} \phi_{t t}=0 .
$$

We see that the quadratic terms vanish, and find that the cubic terms contributing to the $\cos 3 \theta$ term cancel; hence we may take $F_{2}=F_{3}=0$. Then (A 3) reduces to the boundary condition

$$
F_{z}-\left(\omega^{2} / g\right) F=-k^{3} a^{2} \sin \beta F^{3} .
$$

The expression for $\zeta$ from (A 2) may be written as

$$
\zeta=-\frac{1}{g} \phi_{t}+\left\{\frac{1}{2 g^{2}}\left(\phi_{t}^{2}\right)_{z}-\frac{1}{2 g}(\nabla \phi)^{2}\right\}+\left[\frac{1}{2 g^{2}} \phi_{t}(\nabla \phi)^{2}-\frac{1}{4 g^{3}} \phi_{t}\left(\phi_{t}^{2}\right)_{z}\right]_{z} .
$$

From (A 4), with the approximations (A 5) and (A 6) used in the quadratic and cubic terms, we have

$$
\begin{aligned}
\zeta=a \sin \beta\{ & \left\{\cos \theta-\frac{1}{2} k a \cos ^{2} \beta F^{2}+\frac{1}{2} k^{2} a^{2} \sin ^{2} \beta F^{2} \cos 2 \theta\right. \\
& \left.+k^{2} a^{2}\left(\frac{9}{8} \sin ^{4} \beta-\frac{3}{2} \sin ^{2} \beta\right) F^{3} \cos \theta+\frac{3}{8} k^{2} a^{2} \sin ^{4} \beta F^{3} \cos 3 \theta\right\} .
\end{aligned}
$$




\section{REFERENCES}

GUZA, R. T. \& Davis, R. E. 1974 J. Geophys. Res. 79, 1285-1291.

Hanson, E. T. 1926 Proc. Roy. Soc. A 111, 491-529.

MUnk, W., Snodgrass, F. \& CARrier, G. F. 1956 Science, 123, 127-132.

Ostrowskir, L. A. 1967 Sov. Phys., J. Exp. Theor. Phys. 24, 797-800.

OsTrowskII, L. A. 1968 U.R.S.I. Symp. on Electromagnetic Waves VI, Stresa, Italy.

STOKER, J. J. 1957 Water Waves. Interscience.

Stokes, G. G. 1846 Report on recent researches in hydrodynamics. Brit. Ass. Rep. (See also Papers, vol. 1, p. 167. Cambridge University Press.)

UrSell, F. 1952 Proc. Roy. Soc. A 214, 79-97.

Whiтнам, G. B. 1974 Linear and Nonlinear Waves. Interscience. 